\title{
Laboratory diagnosis of SARS-CoV-2: an Update for Clinicians
}

\author{
Imtiyaz A Bhat', Zafar A Shah ${ }^{2}$ \& Shariq R Masoodi ${ }^{3}$ \\ ${ }^{1.2}$ Dept. of Immunology \& Molecular Medicine SKIMS ${ }^{3}$ Dept. of Endocrinology SKIMS
}

SARS-CoV-2 belongs to the genera Beta coronavirus with a spherical or pleomorphic virus enveloped particles. The SARS-CoV-2 has sequence homology with severe acute respiratory syndrome-related coronaviruses (SARSr-CoV) and acts on ACE2 (Angiotensin Converting Enzyme 2) receptor by using its spike proteins. The latter are primed by the enzyme, TMPRSS2 protease to complete this process for its entry into the cells ${ }^{1-3}$. The SARSr-CoV has a singlestranded positive-sense ribonucleic acid (RNA) as a genomic material associated with a nucleoprotein within a capsid comprised of matrix proteins. The envelope bears a club-shaped glycoprotein projection containing haemagglutinin-esterase proteins (HE) also. The size of the Coronaviruses genome is around $(26.4-31.7 \mathrm{~Kb})$, which is one of the largest genomes in RNA known viruses and bears the GC content of approximately 32 to $43 \%$. The viral genome contains distinctive features including a unique $\mathrm{N}$ terminal fragment within the spike proteins. The genome contains a variable number of ORF (611) between the various conserved genes (ORF1ab, spike, envelope, membrane, and nucleocapsid ${ }^{4}$. Two third of the SARS-CoV2 viral RNA is located in the ORF (ORF1a/b) and synthesize polypeptides pp $1 \mathrm{a}$ and $\mathrm{pp} 1 \mathrm{a}$, and 16 non-structural proteins (NSP) are encoded by these ORFs, and the remaining accessory proteins are translated by other ORFs. The genome that encodes essential structural proteins which are important in the pathogenicity of SARS-CoV-2 like spike (S) glycoprotein, small envelope (E) protein, matrix (M) protein, and nucleocapsid $(\mathrm{N})$ protein are translated by the rest of the viral genome ${ }^{5}$.

As reported by the World Health Organization (WHO),

\begin{tabular}{|l|l|}
\hline \multicolumn{2}{|c|}{ Access this article online } \\
\hline
\end{tabular}

SARS-CoV-2 (COVID 19) has infected over 10 million cases from more than 200 countries and has caused 500 thousand deaths worldwide as of 30th June 2020 with experts predicting that the peak is yet to come. With this alarming rate of transmission of SARS-CoV-2 and increasing mortality across the globe, the development of highly sensitive and highly specific investigations should be developed to stop this pandemic from causing more mortalities. Although various detection methods have been developed which will be described in detail but recently the researchers at John Hopkins University estimated that that tested with SAS-CoV-2 in the first four days after infection were 67 percent more likely to test negative, even if they had the virus. When the average patient began displaying symptoms of the virus, the false-negative rate was 38 percent, and the test performed best after eight days of infection but even then had a false negative rate of 20 percent ${ }^{6}$. This high rate of false-negative results during the early days of infection is of great concern for the whole scientific community and needs to be addressed at the earliest. Scientists across the globe are in the process of reducing these false-negative results by designing more sensitive techniques, but despite their best efforts, Kassirer's statement always holds true who states that no diagnostic test can be $100 \%$ accurate $^{7}$.

\section{Analytical issues in-lab diagnosis}

Correspondence:

Dr. Imtiyaz A Bhat, P.hD

Senior Resident

Dept. of Immunology \& Mol. Medicine, SKIMS

Email:imty82@gmail.com

How to cite this article: Bhat IA, Shah ZA, Masoodi S. Laboratory diagnosis of SARS-CoV-2: an Update for Clinicians. JMS 2020; 23(2): 3-8

Received: 2020-06-02 Accepted: 2020-06-15

(ㅇ) (1) This is an open-access article distributed under the terms of the Creative Commons Attribution License (CC BY 4.0), which permits unrestricted use, distribution, and reproduction in any medium, provided the original author and source are credited. 
In the laboratory investigations, usually, four types of conditions can occur during any diagnostic test like Truepositive, False-Positive, False-negative, and True-negative, with true-Positive and True-negative as the desirable situations for any lab to achieve while as False-negative and False positive are the errors which are unavoidable in most of the scenarios ${ }^{8}$. With this background, we will be describing the various diagnostic technique based on different principles that have been designed after the outbreak of COVID-19 and also the various factors that determine their specificity and sensitivity in the detection of SARS-CoV-2. Before discussing the technicalities of these recently designed tests for SARS-CoV-2, the most important factor is pre-analytical errors that occur during the sample collection, sample storage, and sample transportation. In the case of the SARS-CoV-2, the type of specimen is collection plays a significant role in determining the sensitivity of these tests. The most common specimens studied for comparison of positive rates are nasal, pharyngeal, sputum, urine, fiber bronchoscope brush biopsy, blood, and broncho-alveolar lavage fluid (BAL). After studying these specimens from the confirmed cases of SARS-CoV-2 and based on their noninvasive in nature, CDC has recommended the use of nasopharyngeal specimen as a preferred specimen for SARS-CoV-2 detection followed by oropharyngeal, a nasal mid-turbinate specimen (using a flocked tapered swab), an anterior nares (nasal swab) specimen (using a flocked or spun polyester swab) or a nasopharyngeal wash/aspirate or nasal aspirate specimen. The other factors which play a significant role in minimizing the pre-analytical errors are the storage of these specimens in proper viral transport medium vials (VTM) and maintaining the cold chain during the transportation of these samples to the laboratories for analysis. The current diagnostic strategy recommended by the WHO and CDC to identify patients with COVID-19 is to test samples taken from the respiratory tract to assess for the presence of SARSCoV-2 by targeting $\mathrm{m}$ RNA for different genes, SARS-CoV-2 epitopes and screening for antibody against the SARS-CoV-2 antigens. Real-time reverse transcriptasepolymerase chain reaction-based assays are considered the most preferred test for the detection of SARS-CoV-2, while as other diagnostic tests like antigen-based tests and antibody screening are rapidly emerging as an alternative to RT PCR based tests (Figure 1)
Laboratory Diagnostics

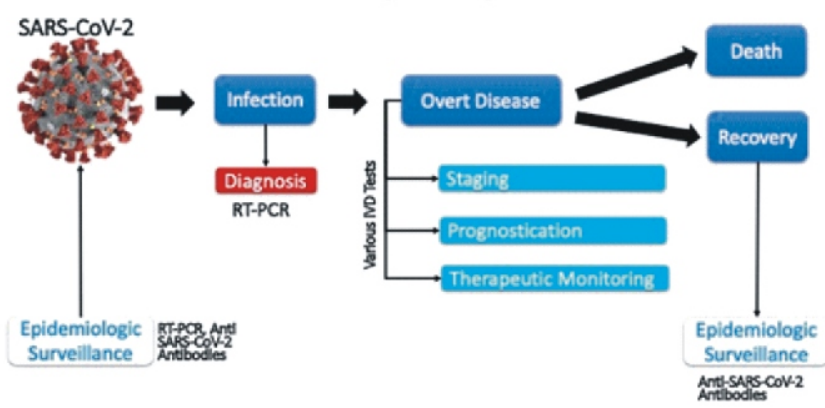

Figure 1: The critical role of Laboratory Medicine in COVID-19 (Modified from Lippi et al [24])

Real-Time Reverse Transcriptase PCR for SARS-CoV-2 detection

Real-time reverse transcriptase-polymerase chain reaction was the first test deployed for the identification of SARSCoV-2 both as a confirmatory diagnosis of symptomatic patients as well as an increased screening of asymptomatic contacts $^{9}$. The development of RT PCR was only possible when the complete genomic sequence of SARS-CoV-2 was published on 23rd January 2020 whichon alignment with the different coronaviruses revealed the 58\% identity among the non-structural proteins as compared to the structural proteins with $48 \%$ identity. The phylogenetic analysis of SARS-CoV-2 among 95 strains isolated from COVID 19 patients around the globe revealed high sequence homology on multiple sequence alignment (99.99\%; range 99.91-100\%) and the amino acid level (99.99\%; range $99.99100 \%$ ) which indicated a low mutation rate for SARS-CoV-2. The sequence homology of the selected conserved target regions from available SARSCoV-2 genomes minimizes the potential cross-reactivity with other coronaviruses, including human coronaviruses and bat-SARS-like coronaviruses ${ }^{3}$. Initially, it was hypothesized that the natural mutation rate and active viral recombination could play an important role in the annealing of designed oligonucleotides and will impair the sensitivity (limit of detection) or might affect the inclusivity i.e., the evolved strains due to these genomic variations will be missed in the RT PCR ${ }^{10,11}$. The study conducted by LuisPeñarrubiaa et al observed that a single genetic variation affects the annealing of different SARS CoV-2 RT panels and recommended the use of multiple targets in the RT PCR could minimize the loss of sensitivity and specificity, however, close monitoring is needed to develop 
Bhat I A et al; Laboratory diagnosis of SARS-CoV-2: an Update for Clinicians

new primers and probes in case of any genetic variability ${ }^{12}$. Based on these findings the protocol for detection for SARSCoV-2 was modified and multiple targets were screened by designing primers and fluorescent probes against genes for RNA-dependent RNA polymerase $(\mathrm{RdRp})$, envelope (E), and nucleocapsid $(\mathrm{N})$ genes of SARS-CoV2. The performance of RT PCR in the detection of SARS-CoV-2 is still around $60-70 \%$ for the nasopharyngeal swab which is the most preferred specimen. With this sensitivity of RT PCR sometimes patients with strong characteristics and evidence of COVID 19 on computed tomography (CT) images are found to be RT Negative. Thus an RT negative result does not exclude the possibility of SARS-CoV-2 infection and should not be the sole criterion for clinical management and patient treatment decisions. The type of specimen has a significant role in detecting the SARS-CoV-2 by RT PCR with nasopharyngeal (63\%) and sputum (72\%) showed better sensitivity than Buccal swab. Wang et al and others have reported the highest sensitivity of $93 \%$ in specimens from bronchoalveolar lavage (BAL) ${ }^{13}$. The amplification of more than one target in the multiplex PCR also helped in minimizing the chances of false-negative results. Post analytical errors like baseline selection, interpretation of CT values, and correlation of amplification plots with the $\mathrm{CT}$ values are also of great significance in result interpretation. The use of NTC, internal negative control, and positive control should play a significant role in minimizing the chances of post-analytical errors. The occurrence of falsepositive results can be minimized by following the proper conditions during sample pooling, nucleic acid extraction, and PCR plate preparation to avoid cross over contamination and carry-over contaminations. The occurrence of analytical errors should never be underestimated in the laboratory medicine in usual circumstances, but the vulnerability of error rate in case of amplification assays like RT PCR is highly magnified due to thehigh workload and severe pressure in a pandemic like COVID 19. Various reports have been published regarding the diagnostic errors due to the exponential peak of positive cases of COVID 19 in many countries; the repercussions of these diagnostic errors are very serious in nature and are unquestionably amplified ${ }^{14-16}$. Because of the several risks to patients in the event of a false-positive result, all clinical laboratories using this test must follow the standard confirmatory testing and reporting guidelines based on their proper public health authorities.

\section{Antibody Testing for SARS-CoV-2}

The use of antibody testing which is cheap, rapid, and easy to perform in comparison to RT PCR holds significant importance in community screening and to find out the development of antibodies in asymptomatic subjects(herd Immunity). There is an urgent need to start serological tests to identify asymptomatic silent infections, evaluate patient immune response, better predict disease progression, and improve our understanding of the epidemiology, including transmission patterns of SARS-CoV-2. Another important role of Serological testing could be an antibody isolation procedure which could play a role in the implementation of convalescent plasma therapy for severely ill COVID-19 patients $^{17}$. At the global market during this COVID pandemic, a wide variety of serological tests enzyme-linked immunosorbent assays, chemiluminescence, and lateral flow assays have been developed ${ }^{17,18}$. This includes a screening of IgA, IgM, and IgG in combination or in isolation at different time intervals for asymptomatic subjects and post-treatment of symptomatic cases. The manufacturing of antibody-based screening kits by around 100 manufacturers are competing for FDA approval but only a few of them have received clearance for mass-scale production. The lack of rigorous validation and evaluation of these assays has not been performed on a large scale and their potential clinical utility is still controversial. The detection of the IgM class of antibody which are usually found during the active course of infection and rises quickly with the onset of infection and decrease very quickly once the infection is neutralized and cannot be a good choice for mass screening in a community ${ }^{19}$. The second class of IgA which is usually present in the mucosal surfaces and plays a vital role in neutralizing microorganisms in the respiratory and gastrointestinal tracts. But like $\operatorname{IgM}, \operatorname{IgA}$ is also are detected during the active infections as early as one day after the SARS-CoV-2 illness. However, the specificity and sensitivity of IgA have been documented until now in the published literature ${ }^{20}$. The pooled results of total antibodies (IgA, IgM, and IgG) specific for SARS-CoV-2 has shown low sensitivity during the first week since onset of symptoms and are below $31 \%$ and subsequently, improved sensitivity rates were seen in the second and 3rd week of infection. IgG \& IgM combination has shown a sensitivity 
Bhat I A et al; Laboratory diagnosis of SARS-CoV-2: an Update for Clinicians

of $30.1 \%$ ( $95 \%$ CI 21.4 to 40.7 ) for 1 to 7 days and the sensitivity subsequently increased to $72.2 \%(95 \% \mathrm{CI} 63.5$ to 79.5) if the sample was collected on 8-14 days postinfection and up to $91 \%((95 \%$ CI 87.0 to 94.4$)$ when the sample was taken on 15-21 days. Beyond 21-35 days the data available is of very small studies, although showing a sensitivity of $96 \%{ }^{19}$. After 35 days of infection, there is insufficient data available. The false-positive rates because of cross-reactivity have been studied extensively and need further research before drawing the final conclusion. In comparison to IgA and IgM class antibodies, IgG class of antibodies is supposed to have a major role in the detection of SARS-Co-V-2 because of its longer shelf life in the serum. The $\operatorname{IgG}$ becomes detectable in the immuno competent after 8 days of getting the SARS-Co-V-2 with more $90 \%$ of the individuals to become seropositive after 14 days of carrying over the virus ${ }^{21}$. The most important contributing factor in determining the sensitivity and specificity of IgG is the source of ELISA kits, according to one standard ELISA kit manufacturer company, the false positive rate of $2.5 \%$ was observed for the diverge range of autoantibodies and $3.4 \%$ cross-reactivity was observed in the sera of influenza vaccine patients ${ }^{20}$. This low false positivity with the IgG class of antibodies would definitely complement the diagnosis but further studies are needed to prove this low false positivity and high sensitivity of $\operatorname{IgG}$ class of antibodies. The sensitivity and specificity of $\operatorname{IgM}$ and IgA have been studied by various studies but the results are not so promising and more research is needed to evaluate their role in the diagnosis of SARS-Co-V- $2^{20}$.

\section{Rapid SARS-CoV-2 antigenic testing}

The SARS-CoV-2 (Antigen) Respi-Strip assay is a new addition to the RT PCR and antibody testing and is based onimmunochroma to graphic principle and has been recently made available for quick diagnosis of SARS-CoV2. The sensitivity of COVID-19 Ag (Antigen) Respi-Strip assay has been proposed to around $60 \%$ and could complement the already available diagnosis of SARS-CoV2. The proposed specificity of antigen-based testing is more than $90 \%$ which would help in taking quick decisions in the management of positive caseswhile the confirmation by RT PCR is only required for negative cases ${ }^{22}$. The principleof rapid COVID-19 Ag Respi-Strip kit by using antibodies against the different epitopes embedded in the surface of
SARS-CoV-2 is now used for diagnostics of COVID 19. This test is qualitativeand easy to perform where the collection of nasopharyngeal swabs done from the person's throat or nose using a swab which is later dipped into a liquid to dissolve the mucus and the virus is released. The liquid containing the dissolved antigen is then applied to the surface with membrane technology with colloidal gold nano particles coated with monoclonal antibodies against conservedepitopes of SARS CoV-2. Another monoclonal antibody that is conjugated to gold nanoparticles is immobilized on to the nitrocellulose membrane. The presence of the SARS CoV-2 is detected by passive diffusion which allows the solubilized conjugate to migrate with the sample and react with the anti-SARS-CoV-2 antibodies immobilized onto the membrane. A control line determines the correct migration of the antigen on the nitrocellulose membrane. The presence of the antigen in the sample is visualized by naked eyes after 15-20 minutes. The proposed sensitivity of $60 \%$ proposed initially for antigen testing has become controversial as only $23.9 \%$ sensitivity was reported by few studies, while one study has reported the sensitivity of around $70 \%$, so more studies should be performed to evaluate the sensitivity and specificity of this tes ${ }^{22,23}$. Although the false negativity rate of around has been detected by a few studies in case of low viral load or low grade of infection. SARS CoV-2 antigen testing can still be of significant importance in filling the gap by providing a fast diagnosis in the clinic, however, a lot more precautions should be taken into considerations in declaring the asymptomatic subjects as negative and RT PCR should be used for the confirm diagnosis.

\section{CONCLUSION}

The sensitivity and specificity of RT PCR in the diagnosis of SARS Co-V-2 still makes a diagnosis of choice for symptomatic cases, however, the use of antigen testing and antibody testing is still the topic of discussion at the local, national and international level. The performance of antigen testing kits and ELISA by various manufacturers needs further research before recommending at the national or international level for mass screening.

\section{References}

1. Mousavizadeh L, Ghasemi S: Genotype and phenotype of COVID-19: Their roles in pathogenesis. Journal of microbiology, immunology, and infection $=$ Wei mian 
Bhat I A et al; Laboratory diagnosis of SARS-CoV-2: an Update for Clinicians

yu gan ranzazhi 2020 .

2. Guo YR, Cao QD, Hong ZS, Tan YY, Chen SD, Jin HJ, Tan KS, Wang DY, Yan Y: The origin, transmission and clinical therapies on coronavirus disease 2019 (COVID-19) outbreak - an update on the status. Military Medical Research 2020, 7(1):11.

3. Lu R, Zhao X, Li J, Niu P, Yang B, Wu H, Wang W, Song $\mathrm{H}$, Huang B, Zhu N et al: Genomic characterisation and epidemiology of 2019 novel coronavirus: implications for virus origins and receptor binding. Lancet 2020, 395(10224):565-574.

4. Wu F, Zhao S, Yu B, Chen YM, Wang W, Song ZG, Hu Y, Tao ZW, Tian JH, Pei YY et al: A new coronavirus associated with human respiratory disease in China. Nature 2020, 579(7798):265-269.

5. Khailany RA, Safdar M, Ozaslan M: Genomic characterisation of a novel SARS-CoV-2. Gene reports 2020:100682.

6. Kucirka LM, Lauer SA, Laeyendecker O, Boon D, Lessler J: Variation in False-Negative Rate of Reverse Transcriptase Polymerase Chain Reaction-Based SARS-CoV-2 Tests by Time Since Exposure. Annals of internal medicine 2020.

7. Kassirer JP: Our stubborn quest for diagnostic certainty. A cause of excessive testing. The New England journal of medicine 1989, 320(22):14891491.

8. Trevethan R: Sensitivity, Specificity, and Predictive Values: Foundations, Pliabilities, and Pitfalls in Research and Practice. Frontiers in public health 2017, 5:307.

9. Shen M, Zhou Y, Ye J, Abdullah Al-Maskri AA, Kang Y, Zeng S, Cai S: Recent advances and perspectives of nucleic acid detection for coronavirus. Journal of pharmaceutical analysis 2020.

10. Lauring AS, Andino R: Quasispecies theory and the behavior of RNA viruses. PLoS pathogens 2010, 6(7):e1001005.

11. Yi H: 2019 novel coronavirus is undergoing active recombination. Clinical infectious diseases: an official publication of the Infectious Diseases Society of America 2020.
12. Penarrubia L, Ruiz M, Porco R, Rao SN, JuanolaFalgarona M, Manissero D, Lopez-Fontanals M, Pareja $\mathrm{J}$ : Multiple assays in a real-time RT-PCR SARS-CoV-2 panel can mitigate the risk of loss of sensitivity by new genomic variants during the COVID-19 outbreak. International Journal of infectious diseases: IJID: official publication of the International Society for Infectious Diseases 2020, 97:225-229.

13. Tahamtan A, Ardebili A: Real-time RT-PCR in COVID-19 detection: issues affecting the results. Expert review of molecular diagnostics 2020, 20(5):453-454.

14. Lippi G, Simundic AM, Plebani M: Potential preanalytical and analytical vulnerabilities in the laboratory diagnosis of coronavirus disease 2019 (COVID-19). Clinical chemistry and laboratory medicine 2020, 58(7):1070-1076.

15. Lippi G, Plebani M: A Six-Sigma approach for comparing diagnostic errors in healthcare-where does laboratory medicine stand? Annals of translational medicine 2018, 6(10):180.

16. Sheridan C: Coronavirus and the race to distribute reliable diagnostics. Nature biotechnology 2020, 38(4):382-384.

17. Yang HS, Racine-Brzostek SE, Lee WT, Hunt D, Yee J, Chen Z, Kubiak J, Cantu M, Hatem L, Zhong E, et al: SARS-CoV-2 antibody characterization in emergency department, hospitalised and convalescent patients by two semi-quantitative immunoassays. Clinica chimica acta; international journal of clinical chemistry 2020, 509:117-125.

18. Udugama B, Kadhiresan P, Kozlowski HN, Malekjahani A, Osborne M, Li VYC, Chen H, Mubareka S, Gubbay JB, Chan WCW: Diagnosing COVID-19: The Disease and Tools for Detection. ACS nano 2020, 14(4):3822-3835.

19. Deeks JJ, Dinnes J, Takwoingi Y, Davenport C, Spijker R, Taylor-Phillips S, Adriano A, Beese S, Dretzke J, Ferrante di Ruffano L, et al: Antibody tests for identification of current and past infection with SARSCoV-2. The Cochrane database of systematic reviews 2020, 6:CD013652.

20. Theel ES, Slev P, Wheeler S, Couturier MR, Wong SJ, 
Bhat I A et al; Laboratory diagnosis of SARS-CoV-2: an Update for Clinicians

Kadkhoda K: The Role of Antibody Testing for SARSCoV-2: Is There One? Journal of Clinical Microbiology 2020:JCM.00797-00720.

21. Amanat F, Stadlbauer D, Strohmeier S, Nguyen THO, Chromikova V, McMahon M, Jiang K, Asthagiri Arunkumar G, Jurczyszak D, Polanco J et al: A serological assay to detect SARS-CoV-2 seroconversion in humans. medRxiv : the preprint server for health sciences 2020 .

22. Blairon L, Wilmet A, Beukinga I, Tré-Hardy M: Implementation of rapid SARS-CoV-2 antigenic testing in a laboratory without access to molecular methods: Experiences of a general hospital. Journal of Clinical Virology 2020, 129:104472.
23. Tre-Hardy M, Wilmet A, Beukinga I, Dogne JM, Douxfils J, Blairon L: Validation of a chemiluminescent assay for specific SARS-CoV-2 antibody. Clinical chemistry and laboratory medicine 2020, 58(8):1357-1364.

24. Lippi G, Plebani M. The Critical Role of Laboratory Medicine During Coronavirus Disease 2019 (COVID19) and Other Viral Outbreaks. Clin Chem Lab Med 2020;58(7):1063-1069. doi: 10.1515/cclm-2020-0240. PMID: 32191623 DOI: $10.1515 / \mathrm{cclm}-2020-0240$ 